\title{
System size dependence of baryon-strangeness correlation in relativistic heavy ion collisions from a multiphase transport model
}

\author{
Dong-Fang Wang (王东方), Song Zhang (张松), ${ }^{*}$ and Yu-Gang Ma (马余刚) ${ }^{\dagger}$ \\ Key Laboratory of Nuclear Physics and Ion-beam Application (MOE), Institute of Modern Physics, \\ Fudan University, Shanghai 200433, China
}

(Received 15 October 2020; accepted 11 January 2021; published 1 February 2021)

\begin{abstract}
The system size dependence of baryon-strangeness (BS) correlation $\left(C_{B S}\right)$ is investigated with a multiphase transport (AMPT) model for various collision systems from ${ }^{10} \mathrm{~B}+{ }^{10} \mathrm{~B},{ }^{12} \mathrm{C}+{ }^{12} \mathrm{C},{ }^{16} \mathrm{O}+{ }^{16} \mathrm{O},{ }^{20} \mathrm{Ne}+{ }^{20} \mathrm{Ne}$, ${ }^{40} \mathrm{Ca}+{ }^{40} \mathrm{Ca},{ }^{96} \mathrm{Zr}+{ }^{96} \mathrm{Zr}$, and ${ }^{197} \mathrm{Au}+{ }^{197} \mathrm{Au}$ at Relativistic Heavy Ion Collider energies $\sqrt{s_{N N}}$ of 200, 39, 27, 20 , and $7.7 \mathrm{GeV}$. Both effects of hadron rescattering and a combination of different hadrons play a leading role in baryon-strangeness correlation. When the kinetic window is limited to absolute rapidity $|y|>3$, these correlations tend to be constant after the final-state interaction, whatever kind of hadron subset we choose based on the AMPT framework. The correlation is found to smoothly increase with baryon chemical potential $\mu_{B}$, corresponding to the collision system or energy from the quark-gluon-plasma-like phase to the hadron-gas-like phase. In addition, the influence of initial nuclear geometric structures of $\alpha$-clustered nuclear collision systems of ${ }^{12} \mathrm{C}+{ }^{12} \mathrm{C}$ as well as ${ }^{16} \mathrm{O}+{ }^{16} \mathrm{O}$ collisions is discussed but the effect is found negligible. The current model studies provide baselines for searching for the signals of the quantum chromodynamics phase transition and critical point in heavy-ion collisions through the BS correlation.
\end{abstract}

DOI: 10.1103/PhysRevC.103.024901

\section{INTRODUCTION}

Relativistic heavy-ion collisions create nuclear matter with sufficient energy density that one expects a quark-gluon plasma (QGP) to form [1-4]. The fundamental challenge remains of how to identify this hot and dense quark matter and fully understand the phase diagram of QGP matter. Lattice QCD calculations have indicated that the transition from hadronic phase to QGP phase is a crossover at zero baryon chemical potential $\left(\mu_{B}=0\right)$ with a transition temperature $T_{c} \approx 166 \mathrm{MeV}[5,6]$. For the finite size system, the transition temperature $T_{c}$ could shift to a value higher than that in an unconstrained space [7]. In an attempt to address these considerations, researchers performed, from 2010 to 2017, a beam-energy scan [8-10] at the BNL Relativistic Heavy Ion Collider (RHIC). One of the promising approaches to probe the QGP phase transition involves fluctuations $[11,12]$.

Theoretical calculations predicted that fluctuations and correlations of conserved charges were distinctly different in the hadronic or QGP phase [13], and they were experimentally accessible to distinguish between these two phases [14]. From

\footnotetext{
*song_zhang@fudan.edu.cn
}

†mayugang@fudan.edu.cn

Published by the American Physical Society under the terms of the Creative Commons Attribution 4.0 International license. Further distribution of this work must maintain attribution to the author(s) and the published article's title, journal citation, and DOI. Funded by $S C O A P^{3}$. experimental analysis of event-by-event fluctuations of net conserved charges like baryon number $(B)$, electric charge $(Q)$, and strangeness $(S)$, in particular, their higher-order cumulants were reported at RHIC $[15,16]$ and the Large Hadron Collider (LHC) [17,18]. One of the event-by-event fluctuations observable was proposed by Koch [19], namely, the baryon-strangeness correlation coefficient,

$$
C_{B S}=-3 \frac{\langle B S\rangle-\langle B\rangle\langle S\rangle}{\left\langle S^{2}\right\rangle-\langle S\rangle^{2}},
$$

where $B$ and $S$ are the net baryon number and net strangeness in one event, respectively. The average value of $B$ and $S$ over a suitable ensemble of events is denoted by $\langle\cdot\rangle$. The $B S$ correlation was considered as a useful tool to characterize if the highly compressed and heated matter created in heavy-ion collisions underwent an ideal QGP phase, strongly coupled QGP phase, or hadronic phase. In previous analyses, several specific models were applied, such as the $(2+1)$ Polyakov quark meson model [20], the hadron resonance gas model [21,22], the URQMD [23-25] model, as well as a multiphase transport (AMPT) model [26], to study the fluctuations and compare them with lattice QCD results [27,28].

Research on small systems has been performed for several years, both experimentally and theoretically [29,30], and several proposals for a system scan (e.g., O + O) [31] have been made to study the possible signals of QGP matter in small systems as well as to investigate the initial state effects on the final state observables [31-33]. We noticed that the ALICE Collaboration reported the enhanced production of multistrange hadrons in high-multiplicity proton-proton collisions [34]. In the same context, we consider that the 
TABLE I. AMPT input parameters and $\left\langle\mathrm{N}_{\text {part }}\right\rangle$ values of different collision systems.

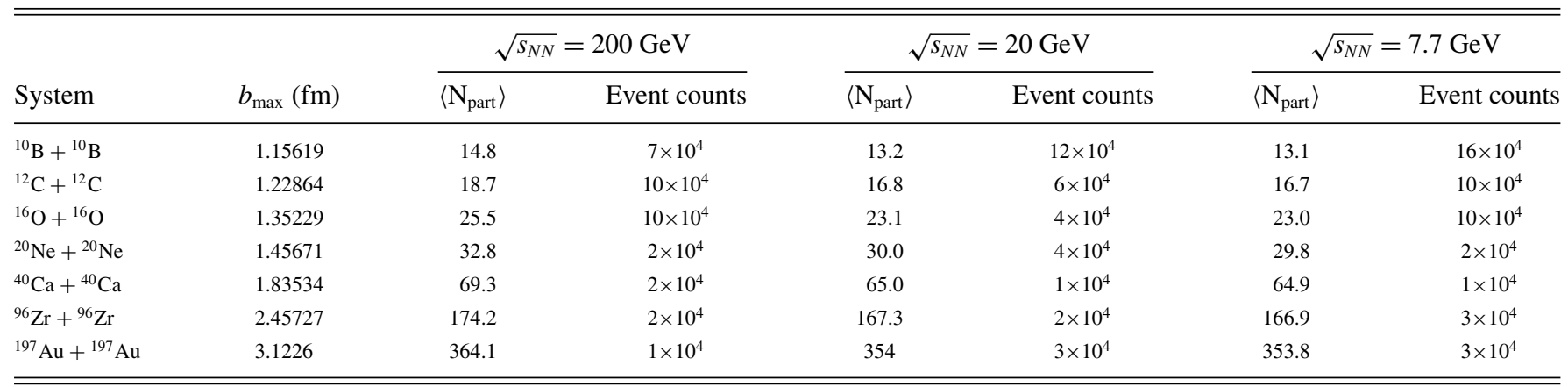

baryon-strangeness correlation which is related to the QGP phase transition may also be sensitive to the fluctuations from small systems to large systems through heavy-ion collisions.

In this work, we adopt a multiphase transport model to study the influence of collision system size on baryonstrangeness correlation $C_{B S}$. By tuning the collision energies of two nuclei, we investigate the energy dependence of the correlation $C_{B S}$. The maximum rapidity acceptance $y_{\max }$ dependence and the influence of initial nuclear geometry structure are also discussed.

The paper is organized as follows. First, in Sec. II, a short introduction to a multiphase transport model and some input parameters are presented and the physical picture of baryonstrangeness correlation is briefly manifested. Next, based on the AMPT model, the dependence of baryon-strangeness correlation as a function of system size, center-of-mass energy, and $y_{\max }$ is discussed in Sec. III. Finally, a brief summary is presented in Sec. IV.

\section{MODEL AND METHODOLOGY}

\section{A. Brief introduction to AMPT model}

AMPT, which is a hybrid dynamic model, is employed to calculate different collision systems. The AMPT model can describe the $p_{T}$ distribution of charged particles [35-38] and their elliptic flow in $\mathrm{Pb}+\mathrm{Pb}$ collisions at $\sqrt{s_{N N}}=2.76$ $\mathrm{TeV}$, as measured through the LHC-ALICE Collaboration. The model includes four main components to describe the relativistic heavy-ion collision process: the initial condition which is simulated using the Heavy Ion Jet Interaction Generator (HIJING) model $[39,40]$, the partonic interaction which is described by Zhang's parton cascade (ZPC) model [41], the hadronization process which proceeds by a Lund string fragmentation or coalescence model, and the hadron rescattering process which is treated by a relativistic transport (ART) model [42]. There are two versions of AMPT: (1) the AMPT version with a string melting mechanism, in which a partonic phase is generated from excited strings in the HIJING model, where a simple quark coalescence model is used to combine the partons into hadrons, and (2) the default AMPT version which only undergoes a pure hadron gas phase. The AMPT model succeeds to describe extensive physics topics for relativistic heavy-ion collisions at the RHIC [43] as well as the LHC [44] energies, e.g., for hadron HanburyBrown and Twiss correlation [45], dihadron azimuthal correlation [46,47], collective flows [48,49], strangeness production $[38,50]$, as well as chiral magnetic effects and so on [51-54]. The details of AMPT can be found in Ref. [43]. In the AMPT model, the impact parameter $b$, demonstrating the transverse distance between the centers of the two collided nuclei, can determine the collision centrality. The number of participants, $\mathrm{N}_{\text {part }}$, is also related to the centrality or impact parameter. We adopt the AMPT parameters as suggested in Ref. [37]. The calculated collision systems and energies, their corresponding maximum impact parameters, $\mathrm{N}_{\text {part }}$, and the number of events are listed in Table I.

\section{B. Baryon-strangeness correlation}

Finding a suitable probe to distinguish QGP matter is the key to understanding the QGP phase transition in relativistic heavy-ion collisions. The correlation coefficient $C_{B S}$, calculated via conserved quantities which are less affected due to uncertainty from hadronization, has an advantage over other probes. Under an ideal QGP assumption, where the basic degrees of freedom are weakly interacting quarks and gluons at high temperature, $\langle S\rangle$ remains zero and $C_{B S}$ can be written as $C_{B S}=-3 \frac{\langle B S\rangle}{\left\langle S^{2}\right\rangle}=1$, noting that the strangeness is only carried by an $s$ quark [19]. However, this feature is different from a hadron gas phase where this coefficient strongly depends on the hadronic environment. Based on an assumption of uncorrelated multiplicities, $C_{B S}$ can be written as [19]

$$
C_{B S} \approx 3 \frac{\langle\Lambda\rangle+\langle\bar{\Lambda}\rangle+\cdots+3\left\langle\Omega^{-}\right\rangle+3\left\langle\bar{\Omega}^{+}\right\rangle}{\left\langle K^{0}\right\rangle+\left\langle\bar{K}^{0}\right\rangle+\cdots+9\left\langle\Omega^{-}\right\rangle+9\left\langle\bar{\Omega}^{+}\right\rangle} .
$$

In actual calculation [19], $C_{B S}$ is expressed as

$$
C_{B S}=-3 \frac{\sum_{n} B^{(n)} S^{(n)}-\frac{1}{N}\left(\sum_{n} B^{(n)}\right)\left(\sum_{n} S^{(n)}\right)}{\sum_{n}\left(S^{(n)}\right)^{2}-\frac{1}{N}\left(\sum_{n} S^{(n)}\right)^{2}},
$$

where $B$ and $S$ denote the net baryon number and net strangeness observed for a given event, respectively, and $N$ is the total number of events. Furthermore, some attention should also be paid to the statistical errors as suggested in Refs. [25,55] (see the Appendix).

\section{RESULTS AND DISCUSSION}

The combination of hadrons would play an important role in the measurement of the baryon-strangeness correlation $C_{B S}$. To investigate this effect, the distribution of net baryons $B$ 



FIG. 1. The correlation between net baryons $B$ and net strangeness $S$ for two different subsets of hadrons in the most central $(0-5 \%){ }^{197} \mathrm{Au}+{ }^{197} \mathrm{Au}$ collisions at $\sqrt{s_{N N}}=200 \mathrm{GeV}$ with the string melting AMPT framework. Case II (a) with and (b) without hadron rescattering, and case I (c) with and (d) without hadron rescattering.

versus net strangeness $S$ is presented in Fig. 1. We chose two combinations of hadrons for baryon-strangeness correlation calculation in this figure: case I, $p+n+\Lambda+\Sigma^{ \pm}+\Xi^{ \pm}+\Omega^{-}+$ $K$, and case II, $p+n+K$, where both the particles and antiparticles were included with kinetic windows $0.1<p_{T}<$ $3.0 \mathrm{GeV} / c$ and $|y|<0.2$. In this figure, we observed that the baryon-strangeness distribution was more concentrated in the center if the effect of hadron rescattering is off, which contributes to stronger correlations. After counting more strange baryons (and antibaryons), the distribution stretches into an elliptical distribution and leads to finite negative correlation. This correlation can be represented as the following:

$$
\rho_{B, S}=\frac{\operatorname{cov}(B, S)}{\sigma_{B} \sigma_{S}}=\frac{\langle(B-\langle B\rangle)(S-\langle S\rangle)\rangle}{\sqrt{\left\langle B^{2}\right\rangle-\langle B\rangle^{2}} \sqrt{\left\langle S^{2}\right\rangle-\langle S\rangle^{2}}} .
$$

The more strange baryons were used, the more negative correlation was present between $B$ and $S$.

We focus on the hadron combination case (case II) for calculating correlations, and also present case I results for comparing effects from different hadron combinations.

Figure 2 shows the system size dependence for all particles (case II) under the effect with or without hadron rescattering in $0-5 \%{ }^{10} \mathrm{~B}+{ }^{10} \mathrm{~B},{ }^{12} \mathrm{C}+{ }^{12} \mathrm{C},{ }^{16} \mathrm{O}+{ }^{16} \mathrm{O},{ }^{20} \mathrm{Ne}+{ }^{20} \mathrm{Ne}$, ${ }^{40} \mathrm{Ca}+{ }^{40} \mathrm{Ca},{ }^{96} \mathrm{Zr}+{ }^{96} \mathrm{Zr}$, and ${ }^{197} \mathrm{Au}+{ }^{197} \mathrm{Au}$ collisions at $\sqrt{s_{N N}}=200 \mathrm{GeV}$ [Fig. 2(a)], $20 \mathrm{GeV}$ [Fig. 2(b)], and $7.7 \mathrm{GeV}$ [Fig. 2(c)] from the AMPT model.

In the case without hadron rescattering where the hadronized system just experienced a partonic phase, the baryon-strangeness correlation $C_{B S}$ keeps constant at 200 and $20 \mathrm{GeV}$ as collision system size increases. At $\sqrt{s_{N N}}=$ 7.7 GeV, $C_{B S}$ almost keeps constant but displays a slightly decreasing trend with system size. As collision energy increases, $C_{B S}$ approaches the value conformed to an ideal QGP assumption $\left(C_{B S}=1\right)$. If hadron rescattering is taken into account the baryon-strangeness correlation $C_{B S}$ exhibits similar behavior at 200 and $20 \mathrm{GeV}$, while it is not completely flat at $7.7 \mathrm{GeV}$. The rescattering process would erase the signal of partonic matter which is consistent with the earlier AMPT study [26].

This dependence is also related to rapidity distribution; thus, the baryon and strangeness yield $d N / d y$ (rapidity density) was also present for ${ }^{197} \mathrm{Au}+{ }^{197} \mathrm{Au}$ collisions at RHIC
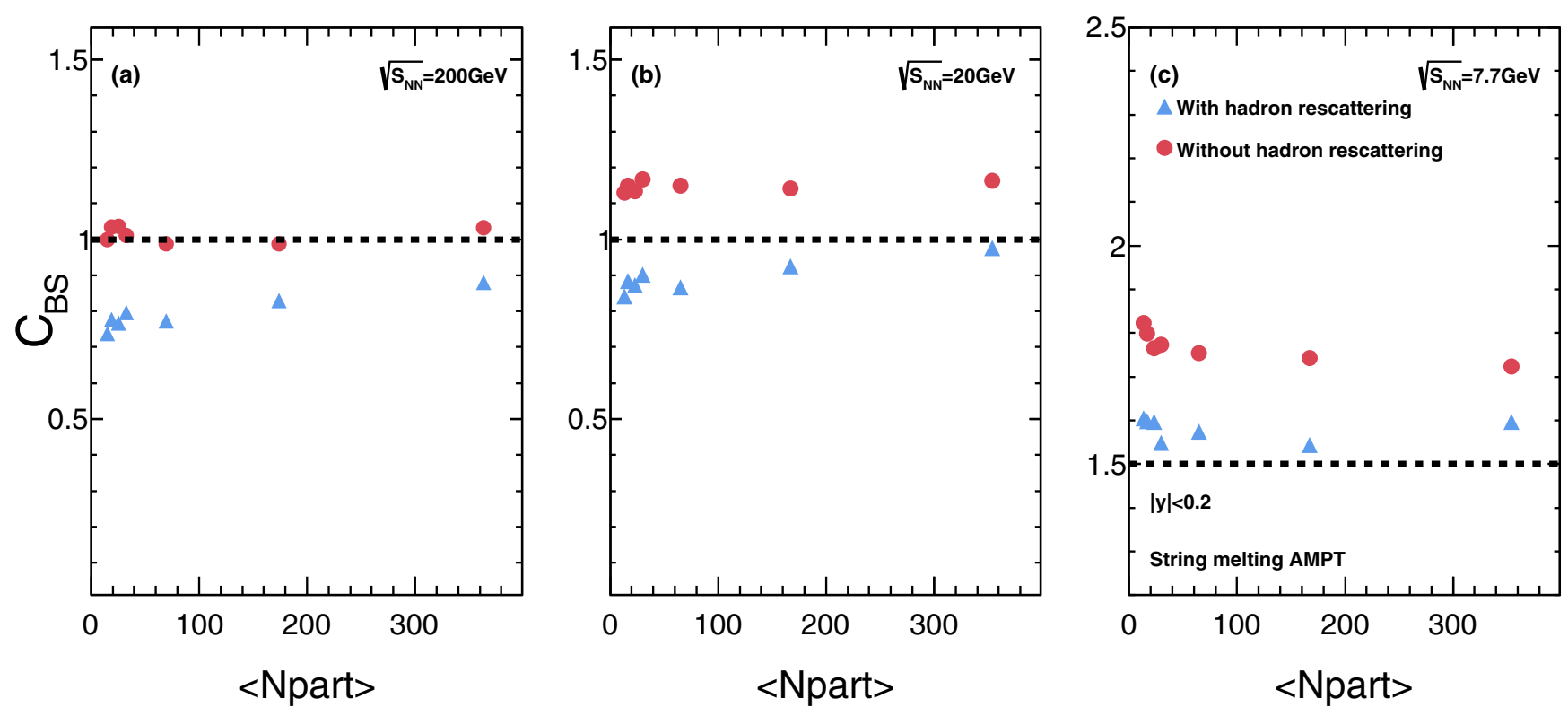

FIG. 2. The baryon-strangeness correlation $C_{B S}$ versus $\left\langle\mathrm{N}_{\text {part }}\right\rangle$ at $\sqrt{s_{N N}}=200,20$, and $7.7 \mathrm{GeV}$ in the most central collisions $(0-5 \%)$ of ${ }^{10} \mathrm{~B}+{ }^{10} \mathrm{~B},{ }^{12} \mathrm{C}+{ }^{12} \mathrm{C},{ }^{16} \mathrm{O}+{ }^{16} \mathrm{O},{ }^{20} \mathrm{Ne}+{ }^{20} \mathrm{Ne},{ }^{40} \mathrm{Ca}+{ }^{40} \mathrm{Ca},{ }^{96} \mathrm{Zr}+{ }^{96} \mathrm{Zr}$, and ${ }^{197} \mathrm{Au}+{ }^{197} \mathrm{Au}$ systems at RHIC energies $\sqrt{s_{N N}}=($ a) 200, (b) 20, and (c) $7.7 \mathrm{GeV}$ in the string melting AMPT framework. 

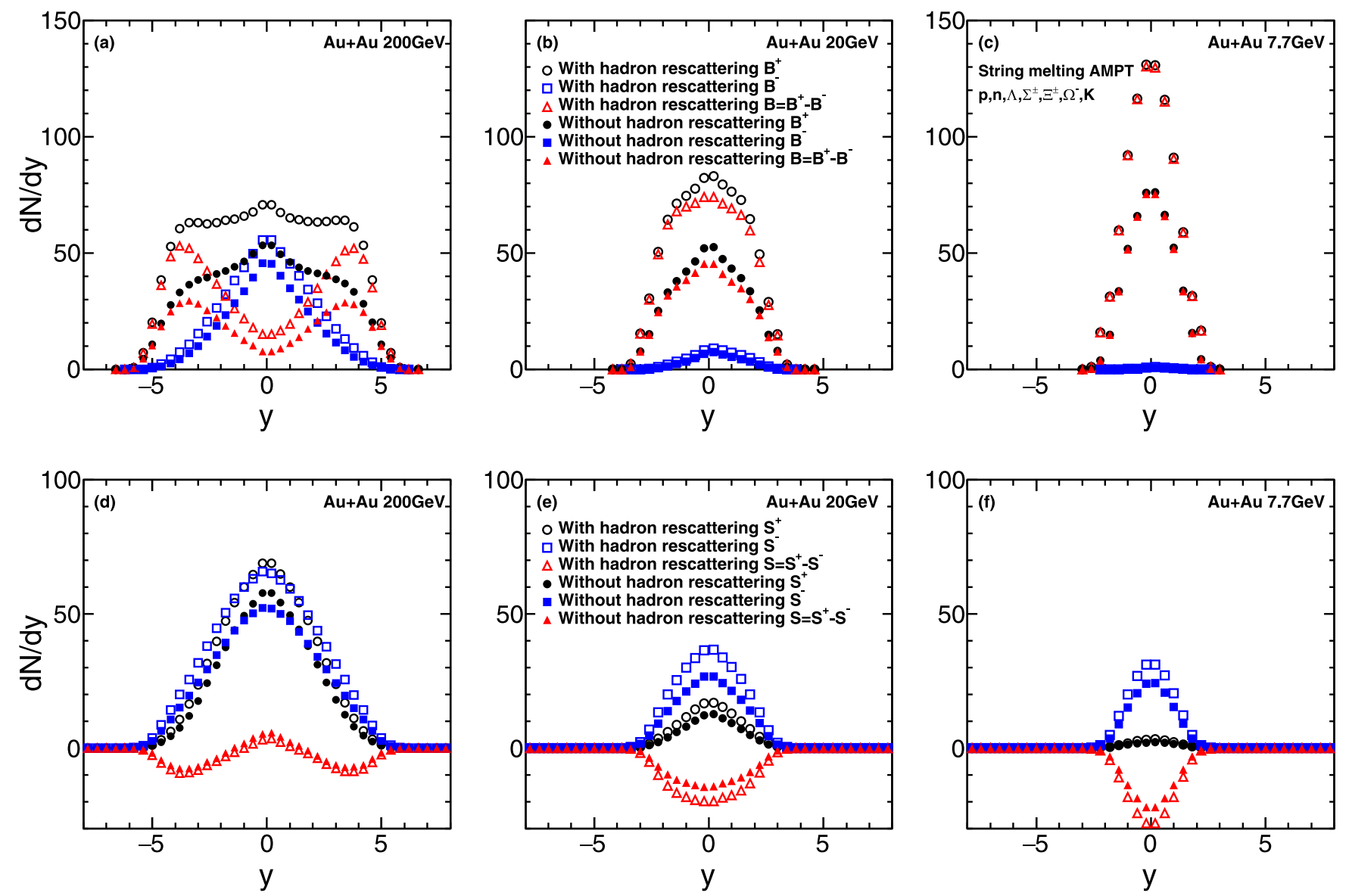

FIG. 3. The AMPT results of positive baryon (strangeness) $B^{+}\left(S^{+}\right)$, negative baryon (strangeness) $B^{-}\left(S^{-}\right)$, and net baryon (net strangeness) $B(S) d N / d y$ for identified particles, namely, $p, n, \Lambda, \Sigma^{ \pm}, \Xi^{ \pm}, \Omega^{-}$, and $K$ in ${ }^{197} \mathrm{Au}+{ }^{197} \mathrm{Au}$ collisions at RHIC energies $\sqrt{s_{N N}}=$ (a, d) 200, (b, e) 20, and (c, f) $7.7 \mathrm{GeV}$ based on the string melting AMPT framework. The meaning of different symbols is illustrated in the insets of (b) and (e). Here the kinematics window is $|y|<0.2$.

energies $\sqrt{s_{N N}}=200,20$, and $7.7 \mathrm{GeV}$ based on the string melting AMPT model, as shown in Fig. 3. We observed that the rapidity distribution of net baryons $B$ becomes more concentrated in the middle rapidity when collision energy decreases as presented in Figs. 3(a)-3(c). At $\sqrt{s_{N N}}=20$ and $7.7 \mathrm{GeV}$, the lower collision energy makes positive baryon $B^{+}$ much larger than negative baryon $B^{-}$(almost contributed by antiprotons). After the hadron rescattering process, as a result of the contribution of decay particles, the net baryon $B$ value is a little higher than the values without hadron rescattering. The non-Gaussian distribution of the $B$ rapidity at $200 \mathrm{GeV}$ was also found in Ref. [56].

Figures 3(e) and 3(f) display the rapidity distribution of net strangeness $S$ at $\sqrt{s_{N N}}=20$ and $7.7 \mathrm{GeV}$, respectively, where $S$ is always negative. However, $S$ turns positive at $200 \mathrm{GeV}$ in mid-rapidity as shown in Fig. 3(d). As energy increases, the rapidity density of $B$ decreases in the chosen region, and the baryon-strangeness correlation $C_{B S}$ is closer to 1 , manifesting that the system is close to the QGP state. The more net baryons $B$ the collision system has, the closer the state would be to the hadron gas phase with a larger value of $C_{B S}$.

As energy decreases, the rapidity densities of net baryons $B$ and net strangeness $S$ grow a more sharp peak as plotted in Fig. 3. Therefore, the final result of $C_{B S}$ will be affected greatly by a slight change of the dynamic window size. Consequently, we could draw the conclusion that $C_{B S}$ is also affected by kinetic windows due to this nonflat rapidity distribution.

We also presented $C_{B S}$ as a function of the rapidity acceptance range $y_{\max }$ in $\mathrm{Au}+\mathrm{Au}$ collisions at $\sqrt{s_{N N}}=$ $200 \mathrm{GeV}$ by the AMPT model. As manifested in Fig. 4, we observed two different $y_{\max }$ dependencies based on the two different combinations of hadrons. The correlation coefficient tends to increase with $y_{\max }$ in the case II hadron combination. However, when the case I hadron combination is chosen, the coefficient tends to decrease. Although the hadron combinations were different, $C_{B S}$ goes asymptotically to a constant as $y_{\max }>3$. Additionally, the choice of hadron combination has no effect on the result at large $y_{\max }$ at the hadron rescattering stage as a consequence of conserved quantities of baryon number and strangeness. In a previous study [19], the correlation coefficient $C_{B S}$ first increased with $y_{\max }$ and reached a maximum value at a certain $y_{\max }$ before it dropped to zero.

To understand this phenomenon, Figs. 5(a) and 5(b) display the maximum rapidity acceptance $\left(|y|<y_{\max }\right)$ dependence of the numerator $\left(C_{11}^{B S}=\langle B S\rangle-\langle B\rangle\langle S\rangle\right)$ and the denominator $\left(C_{2}^{S}=\left\langle S^{2}\right\rangle-\langle S\rangle^{2}\right)$ of $C_{B S}$ in ${ }^{197} \mathrm{Au}+{ }^{197} \mathrm{Au}$ collisions at $\sqrt{s_{N N}}=200 \mathrm{GeV}$ with the string melting AMPT model, respectively. In the case II hadron combination, both the $-3 C_{11}^{B S}$ 


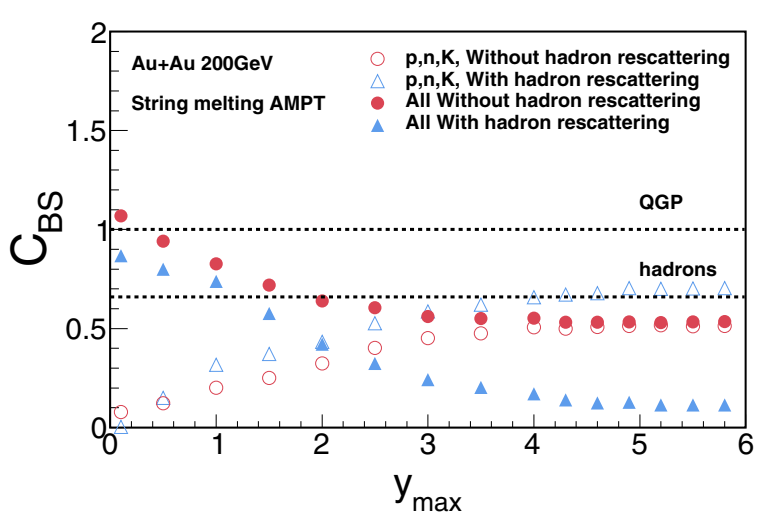

FIG. 4. The maximum rapidity acceptance $\left(|y|<y_{\max }\right)$ dependence of the correlation coefficient $C_{B S}$ in ${ }^{197} \mathrm{Au}+{ }^{197} \mathrm{Au}$ collisions at $\sqrt{s_{N N}}=200 \mathrm{GeV}$ in the string melting AMPT framework. Two different subsets of hadrons are adopted to show different dependencies. The two dashed lines indicate the theoretical estimate of simple QGP $\left(C_{B S}=1\right)$ and hadron gas $\left(C_{B S}=0.66\right)$ at chemical freeze-out conditions of $T=170 \mathrm{MeV}$ and $\mu_{b}=0$, respectively.

and the $C_{2}^{S}$ gradually tend to a constant value as $y_{\max }$ increases, as shown in Figs. 5(a) and 5(b), respectively. However, in the case I hadron combination, the $-3 C_{11}^{B S}$ increases with $y_{\max }$ and then drops to a constant value with the hadron rescattering process. Thus, the value of $C_{11}^{B S}$ is the dominant factor affecting the correlation coefficient $C_{B S}$.

The $C_{B S}$ calculated in this model is plotted in Fig. 6(a) at $\sqrt{s_{N N}}=11.5,20,27,39$, and $200 \mathrm{GeV}$ in ${ }^{197} \mathrm{Au}+{ }^{197} \mathrm{Au}$ central collisions and presented strong energy dependence. As energy increases, $C_{B S}$ goes down to 0.8 at the top RHIC energy. This result was also below the expected value for an ideal QGP phase which was mentioned in Ref. [24]. Figure 6(b) shows $C_{B S}$ as a function of baryon chemical potential $\mu_{B}$ at chemical freeze-out for $\sqrt{s_{N N}}=200 \mathrm{GeV}$ collision systems, where $\mu_{B}$ was extracted based on the thermal model as given in our previous paper [57]. At a given collision energy, $\mu_{b}$ increases with system size, and a similar trend also appears in Fig. 2(a). The correlation coefficient $C_{B S}$ with the hadron rescattering process slightly increases with $\mu_{b}$, which is consistent with the previous conclusion [19]. Meanwhile, the collision energy dependence of $C_{B S}$ is displayed in
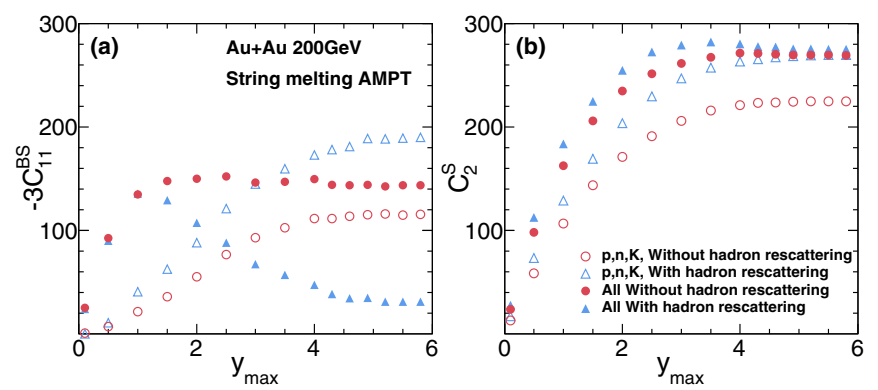

FIG. 5. The maximum rapidity acceptance $\left(|y|<y_{\max }\right)$ dependence of (a) the numerator $\left(C_{11}^{B S}\right)$ and (b) the denominator $\left(C_{2}^{S}\right)$ (b) of $C_{B S}$ in ${ }^{197} \mathrm{Au}+{ }^{197} \mathrm{Au}$ collisions at $\sqrt{s_{N N}}=200 \mathrm{GeV}$ with the string melting AMPT framework. Two different subsets of hadrons are adopted to show different dependencies.

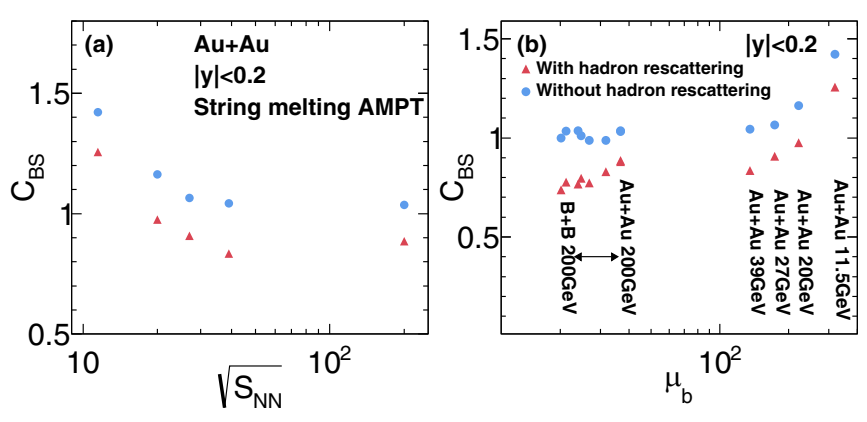

FIG. 6. (a) The correlation coefficient $C_{B S}$ in the most central $(0-5 \%){ }^{197} \mathrm{Au}+{ }^{197} \mathrm{Au}$ collisions is shown as a function of $\sqrt{s_{N N}}$. (b) The correlation coefficient $C_{B S}$ for a hadron gas at freeze-out is shown as a function of the baryon chemical potential $\mu_{B}$ in the most central $(0-5 \%)$ collision at different collision systems and energy. For the $C_{B S}$ system scan, we chose ${ }^{10} \mathrm{~B}+{ }^{10} \mathrm{~B},{ }^{12} \mathrm{C}+{ }^{12} \mathrm{C},{ }^{16} \mathrm{O}+{ }^{16} \mathrm{O}$, ${ }^{20} \mathrm{Ne}+{ }^{20} \mathrm{Ne},{ }^{40} \mathrm{Ca}+{ }^{40} \mathrm{Ca},{ }^{96} \mathrm{Zr}+{ }^{96} \mathrm{Zr}$, and ${ }^{197} \mathrm{Au}+{ }^{197} \mathrm{Au}$ collisions at $\sqrt{s_{N N}}=200 \mathrm{GeV}$. For the $C_{B S}$ energy scan, the choice of energy is $\sqrt{s_{N N}}=11.5,20,27,39$, and $200 \mathrm{GeV}$.

Fig. 6(b). For a given system, there were more net baryons in the collision system as energy decreased, leading to the $C_{B S}$ enhancement. The correlation coefficient presents a smooth baryon chemical potential dependence if collision systems and collision energies are characterized by the potential.

Finally, we investigated the possible effect of $\alpha$-clustering structure of light nuclei on the correlation coefficient $C_{B S}$. Some previous works proposed that the signatures of $\alpha$ clustering structure in light nuclei could be observed via heavy-ion collisions at ultrarelativistic energies [32,58-66]. In this context, we examined the influence of the fluctuation of initial nuclear geometric structure on the correlation coefficient $C_{B S}$. To this end, the nucleon distribution might be considered as a three- $\alpha$ clustering triangle structure for ${ }^{12} \mathrm{C}$ and four- $\alpha$ clustering tetrahedron structure for ${ }^{16} \mathrm{O}$ in the present study. Figure 7 demonstrates $\alpha$-clustering effects on



FIG. 7. The correlation coefficient $C_{B S}$ as a function of the number of participants, $\left\langle\mathrm{N}_{\mathrm{part}}\right\rangle$, which are obtained from different center-of-mass energies at $\sqrt{s_{N N}}=10,200$, and $6370 \mathrm{GeV}$ in the most central collisions (impact parameter $b=0$ ) of ${ }^{12} \mathrm{C}+{ }^{12} \mathrm{C}$ and ${ }^{16} \mathrm{O}+{ }^{16} \mathrm{O}$ systems. 


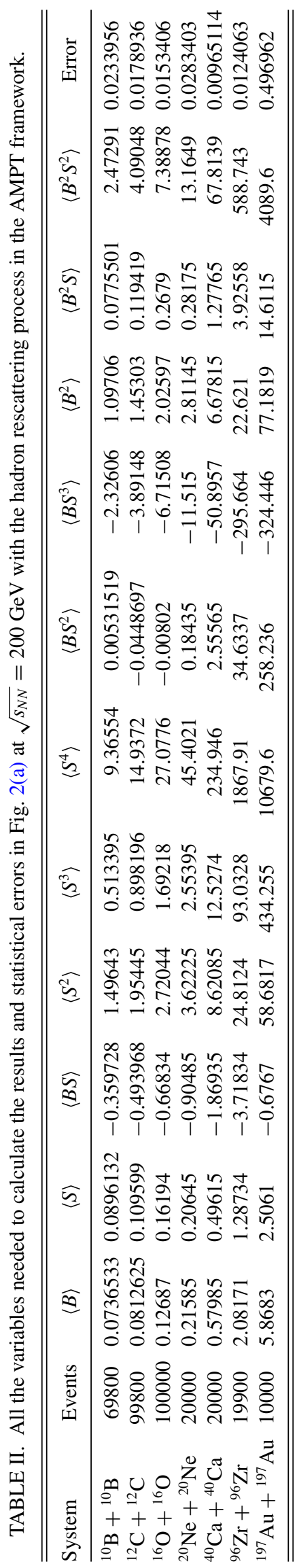

$C_{B S}$ for ${ }^{12} \mathrm{C}+{ }^{12} \mathrm{C}$ as well as ${ }^{16} \mathrm{O}+{ }^{16} \mathrm{O}$ collisions at $\sqrt{s_{N N}}$ $=10,200$, and $6370 \mathrm{GeV}$, respectively. These collision systems with different $\alpha$-clustering structure and energies were labeled by $\left\langle\mathrm{N}_{\text {part }}\right\rangle$. The results show nearly no difference visibly between the Woods-Saxon nucleon distribution and the $\alpha$-clustering structures via $C_{B S}$ coefficient. It implies that the baryon-strangeness correlation was insensitive to initial nucleon distribution, which could in turn help to isolate other ingredients for affecting $C_{B S}$, such as the hadron rescattering effect as discussed in this work.

\section{SUMMARY}

In summary, we studied the system and energy dependence of the baryon-strangeness correlation coefficient in the framework of the AMPT model. The hadron rescattering process partly weakens the baryon-strangeness correlation as expected, and weak decay contributions for strangeness or the count of baryons might have an effect on the final results, which need to be further investigated. The combination of different hadrons additionally affects the results significantly. In addition, it was found that when the maximum rapidity acceptance $y_{\max }>3$, the baryon-strangeness coefficient is independent of the combination of different hadrons in the final state based on the AMPT model. The correlation coefficient could be grouped if the collision systems and collision energies were characterized by the baryon chemical potential. In addition, we investigated the effect of initial nucleon distribution for light nuclei, specifically with either the Woods-Saxon nucleon distribution or the $\alpha$-clustering structure for ${ }^{12} \mathrm{C}$ and ${ }^{16} \mathrm{O}$ nuclei, on the final baryon-strangeness correlation results but found negligible effect. These AMPT model studies provide baselines for searching for the signals of the QCD phase transition and critical point in heavy-ion collisions through the BS correlation.

\section{ACKNOWLEDGMENTS}

This work was supported in part by the Guangdong Major Project of Basic and Applied Basic Research No. 2020B0301030008, the Strategic Priority Research Program of CAS under Grant No. XDB34000000, the National Natural Science Foundation of China under Contracts No. 11875066, No. 11890710, No. 11890714, No. 11925502, and No. 11961141003 , the National Key R\&D Program of China under Grants No. 2016YFE0100900 and No. 2018YFE0104600, and the Key Research Program of Frontier Sciences of the CAS under Grant No. QYZDJ-SSW-SLH002.

\section{APPENDIX A: OBSERVABLE}

The joint cumulant of several random variables $X_{1}, \ldots, X_{n}$ is defined by a similar cumulant generating function,

$$
K\left(t_{1}, t_{2}, \ldots, t_{n}\right)=\log E\left(e^{\sum_{j=1}^{n} t_{j} X_{j}}\right) .
$$

A consequence is that

$$
\kappa\left(X_{1}, \ldots, X_{n}\right)=\sum_{\pi}(|\pi|-1) !(-1)^{|\pi|-1} \prod_{B \in \pi} E\left(\prod_{i \in B} X_{i}\right),
$$


where $\pi$ runs through the list of all partitions of $\{1, \ldots, n\}$, $B$ runs through the list of all blocks of the partition $\pi$, and $|\pi|$ is the number of parts in the partition. In this analysis, we use $B$ and $S$ to represent the net baryon number and the net strangeness in one event, respectively. The deviation of $B$ and $S$ from their mean values are expressed by $\delta B=B-\langle B\rangle$ and $\delta S=S-\langle S\rangle$, respectively. As mentioned above, we use $\langle\cdot\rangle$ to represent expected value. According to Eq. (2),

$$
\begin{aligned}
& C(\delta B, \delta S)=\langle\delta B \delta S\rangle=\langle B S\rangle-\langle B\rangle\langle S\rangle, \\
& C(\delta S, \delta S)=\langle\delta S \delta S\rangle=\left\langle S^{2}\right\rangle-\langle S\rangle^{2} .
\end{aligned}
$$

Thus, the baryon-strangeness correlation coefficient is

$$
C_{B S}=-3 \frac{C(\delta B, \delta S)}{C(\delta S, \delta S)}=-3 \frac{\langle B S\rangle-\langle B\rangle\langle S\rangle}{\left\langle S^{2}\right\rangle-\langle S\rangle^{2}} .
$$

\section{APPENDIX B: THE STATISTICAL ERROR OF $C_{B S}$}

In the Appendix of Ref. [25], the authors showed in detail how to calculate the statistical uncertainty by way of the covariance of the multivariate moments. According to the definition of the covariance,

$$
\operatorname{cov}\left(f_{i, j}, f_{k, h}\right)=\frac{1}{N}\left(f_{i+k, j+h}-f_{i, j} f_{k, h}\right),
$$

higher-order terms must be introduced for calculating the covariance. So we give all the items necessary for calculating the error throughout Table II. From the equation we know the error is proportional to $1 / \sqrt{N}$; however, the corresponding event statistics we use are relatively small, and the statistical errors of results would be large.
[1] J. Adams et al., Nucl. Phys. A 757, 102 (2005).

[2] B. B. Back et al., Nucl. Phys. A 757, 28 (2005).

[3] K. Adcox et al., Nucl. Phys. A 757, 184 (2005).

[4] I. Arsene et al., Nucl. Phys. A 757, 1 (2005).

[5] Y. Aoki, S. Borsnyi, S. Drr, Z. Fodor, S. D. Katz, S. Krieg, and K. Szabo, J. High Energy Phys. 06 (2009) 088.

[6] A. Bazavov, T. Bhattacharya, M. Cheng, C. DeTar, H. T. Ding, S. Gottlieb, R. Gupta, P. Hegde, U. M. Heller, F. Karsch et al. (HotQCD Collaboration), Phys. Rev. D 85, 054503 (2012).

[7] Z. Han, B. Chen, and Y. Liu, Chin. Phys. Lett. 37, 112501 (2020).

[8] K. H. Ackermann et al., Nucl. Instrum. Methods Phys. Res., Sect. A 499, 624 (2003).

[9] M. Aggarwal et al. (STAR Collaboration), arXiv:1007.2613.

[10] L. Adamczyk et al. (STAR Collaboration), Phys. Rev. C 96, 044904 (2017).

[11] V. Koch, arXiv:0810.2520.

[12] S. Jeon and V. Koch, arXiv:hep-ph/0304012.

[13] X. F. Luo and N. Xu, Nucl. Sci. Tech. 28, 112 (2017).

[14] A. Adare et al. (PHENIX Collaboration), Phys. Rev. C 93, 011901 (2016).

[15] A. Chatterjee, PoS (CORFU2018), 164 (2019).

[16] L. Adamczyk et al. (STAR Collaboration), Phys. Rev. Lett. 113, 092301 (2014).

[17] B. Friman, F. Karsch, K. Redlich, and V. Skokov, Euro. Phys. J. C 71, 1694 (2011).

[18] A. Rustamov, Nucl. Phys. A 967, 453 (2017).

[19] V. Koch, A. Majumder, and J. Randrup, Phys. Rev. Lett. 95, 182301 (2005).

[20] S. Chatterjee and K. A. Mohan, Phys. Rev. D 86, 114021 (2012).

[21] A. Bazavov, T. Bhattacharya, C. E. DeTar, H.-T. Ding, S. Gottlieb, R. Gupta, P. Hegde, U. M. Heller, F. Karsch, E. Laermann et al. (HotQCD Collaboration), Phys. Rev. D 86, 034509 (2012).

[22] A. Bhattacharyya, S. Das, S. K. Ghosh, R. Ray, and S. Samanta, Phys. Rev. C 90, 034909 (2014).

[23] A. Chatterjee, S. Chatterjee, T. K. Nayak, and N. R. Sahoo, J. Phys. G 43, 125103 (2016).

[24] S. Haussler, S. Scherer, and M. Bleicher, in Quark Confinement and the Hadron Spectrum VII: 7th Conference on Quark Confinement and the Hadron Spectrum (QCHS7), edited by J.
Emilio, F. T. Ribeiro, N. Brambilla, A. Vairo, K. Maung, and G. M. Prosperi, AIP Conf. Proc. No. 892 (AIP, New York, 2007), pp. 372-376.

[25] Z. Z. Yang, X. F. Luo, and B. Mohanty, Phys. Rev. C 95, 014914 (2017).

[26] F. Jin, Y. G. Ma, G. L. Ma, J. H. Chen, S. Zhang, X. Z. Cai, H. Z. Huang, J. Tian, C. Zhong, and J. X. Zuo, J. Phys. G 35, 044070 (2008).

[27] R. V. Gavai and S. Gupta, Phys. Rev. D 73, 014004 (2006).

[28] A. Bazavov, H. T. Ding, P. Hegde, O. Kaczmarek, F. Karsch, E. Laermann, Y. Maezawa, S. Mukherjee, H. Ohno, P. Petreczky, C. Schmidt, S. Sharma, W. Soeldner, and M. Wagner, Phys. Rev. Lett. 113, 072001 (2014).

[29] C. Loizides, Nucl. Phys. A 956, 200 (2016).

[30] J. D. Orjuela Koop, R. Belmont, P. Yin, and J. L. Nagle, Phys. Rev. C 93, 044910 (2016).

[31] M. Sievert and J. Noronha-Hostler, Phys. Rev. C 100, 024904 (2019).

[32] S. Huang, Z. Chen, W. Li, and J. Jia, Phys. Rev. C 101, 021901(R) (2020).

[33] S. Zhang, Y. G. Ma, G. L. Ma, J. H. Chen, Q. Y. Shou, W. B. He, and C. Zhong, Phys. Lett. B 804, 135366 (2020).

[34] J. Adam et al. (ALICE Collaboration), Nat. Phys. 13, 535 (2017).

[35] J. Xu and C. M. Ko, Phys. Rev. C 83, 034904 (2011).

[36] S. Pal and M. Bleicher, Phys. Lett. B 709, 82 (2012).

[37] Y. J. Ye, J. H. Chen, Y. G. Ma, S. Zhang, and C. Zhong, Chin. Phys. C 41, 084101 (2017).

[38] X. H. Jin, J. H. Chen, Y. G. Ma, S. Zhang, C. J. Zhang, and C. Zhong, Nucl. Sci. Tech. 29, 54 (2018).

[39] X. N. Wang and M. Gyulassy, Phys. Rev. D 44, 3501 (1991)

[40] M. Gyulassy and X. N. Wang, Comput. Phys. Commun. 83, 307 (1994).

[41] B. Zhang, Comput. Phys. Commun. 109, 193 (1998).

[42] B. A. Li and C. M. Ko, Phys. Rev. C 52, 2037 (1995).

[43] Z. W. Lin, C. M. Ko, B. A. Li, B. Zhang, and S. Pal, Phys. Rev. C 72, 064901 (2005).

[44] G. L. Ma and Z. W. Lin, Phys. Rev. C 93, 054911 (2016).

[45] Z. W. Lin, C. M. Ko, and S. Pal, Phys. Rev. Lett. 89, 152301 (2002)

[46] G. L. Ma et al., Phys. Lett. B 641, 362 (2006). 
[47] H. Wang, J. H. Chen, Y. G. Ma, and S. Zhang, Nucl. Sci. Tech. 30, 185 (2019).

[48] B. I. Abelev et al. (STAR Collaboration), Phys. Rev. Lett. 101, 252301 (2008).

[49] A. Bzdak and G. L. Ma, Phys. Rev. Lett. 113, 252301 (2014).

[50] X. H. Jin, J. H. Chen, Z. W. Lin, G. L. Ma, Y. G. Ma, and S. Zhang, Sci. China Phys. Mech. Astron. 62, 11012 (2019).

[51] X. L. Zhao, G. L. Ma, and Y. G. Ma, Phys. Lett. B 792, 413 (2019).

[52] Y. C. Liu and X. G. Huang, Nucl. Sci. Tech. 31, 56 (2020).

[53] F. Q. Wang and J. Zhao, Nucl. Sci. Tech. 29, 179 (2018).

[54] Z. W. Xu, S. Zhang, Y. G. Ma, J. H. Chen, and C. Zhong, Nucl. Sci. Tech. 29, 186 (2018).

[55] F. L. Xiao, J. Phys. G 39, 025008 (2012).

[56] Y. F. Lin, L. Z. Chen, and Z. M. Li, Phys. Rev. C 96, 044906 (2017).

[57] D. F. Wang, S. Zhang, and Y. G. Ma, Phys. Rev. C 101, 034906 (2020).
[58] W. Broniowski and E. Ruiz Arriola, Phys. Rev. Lett. 112, 112501 (2014).

[59] W. B. He, Y. G. Ma, X. G. Cao, X. Z. Cai, and G. Q. Zhang, Phys. Rev. Lett. 113, 032506 (2014).

[60] P. Bożek, W. Broniowski, E. R. Arriola, and M. Rybczyński, Phys. Rev. C 90, 064902 (2014).

[61] S. Zhang, Y. G. Ma, J. H. Chen, W. B. He, and C. Zhong, Phys. Rev. C 95, 064904 (2017).

[62] S. Zhang, Y. G. Ma, J. H. Chen, W. B. He, and C. Zhong, Eur. Phys. J. A 54, 161 (2018).

[63] Y. L. Cheng, S. Zhang, Y. G. Ma, J. H. Chen, and C. Zhong, Phys. Rev. C 99, 054906 (2019).

[64] S. H. Lim, J. Carlson, C. Loizides, D. Lonardoni, J. E. Lynn, J. L. Nagle, J. D. Orjuela Koop, and J. Ouellette, Phys. Rev. C 99, 044904 (2019).

[65] J. J. He, S. Zhang, Y. G. Ma, J. H. Chen, and C. Zhong, Eur. Phys. J. A 56, 52 (2020).

[66] L. Ma, Y. G. Ma, and S. Zhang, Phys. Rev. C 102, 014910 (2020). 